Cite this: Phys. Chem. Chem. Phys., 2012, 14, 14523-14533

\title{
Bicellar systems to modify the phase behaviour of skin stratum corneum lipids
}

\author{
Gelen Rodríguez, ${ }^{* a}$ Mercedes Cócera,${ }^{a}$ Laia Rubio, ${ }^{a}$ Cristina Alonso, ${ }^{a}$ \\ Ramon Pons, ${ }^{a}$ Christophe Sandt, ${ }^{b}$ Paul Dumas, ${ }^{b}$ Carmen López-Iglesias, ${ }^{c}$ \\ Alfons de la $\mathrm{Maza}^{a}$ and Olga López ${ }^{a}$
}

Received 21st April 2012, Accepted 28th August 2012

DOI: $10.1039 / \mathrm{c} 2 \mathrm{cp} 42421 \mathrm{e}$

Bicellar systems are a fascinating category of versatile lipid assemblies that comprise bilayered disk-shaped nanoaggregates formed in water by long and short alkyl chain phospholipids. Bicelles bridge the gap between micelles and lipid vesicles by combining the attractive properties of both systems. These structures have recently been proposed in dermatological, cosmetic and pharmaceutical applications. Two new binary bicellar systems composed of cholesterol sulphate (SCHOL) and long-chain phospholipids (dimyristoyl-phosphatidylcholine, DMPC, or dipalmitoylphosphatidylcholine, DPPC) are characterised herein by differential scanning calorimetry, fluorescence spectroscopy, X-ray scattering and microscopy. Additionally, a comparative study on skin treated with the new SCHOL systems (DMPC/SCHOL and DPPC/SCHOL) and classic DHPC systems (DMPC/DHPC and DPPC/DHPC) was performed. These studies were conducted to determinate how deeply bicelles penetrate into the skin and the extension of their effect on the phase behaviour of stratum corneum (SC) lipids using attenuated total reflectance-Fourier transform infrared spectroscopy and two-photon excitation fluorescence microscopy. Our results show that SCHOL modified the typical discoidal morphology and the phase behaviour of the systems, inducing coexistence of two phases, liquid-ordered and ripple phases. The effect of the systems on SC lipids depends on their composition and is related to the fluidity of the SC lipid alkyl chains. Thus, systems with DMPC induced more disorder in SC lipids than systems with DPPC, and SCHOL did not modify the lipid arrangement. Perdeuterated systems in the infrared spectroscopy technique supported a different distribution in the tissue for every system. DMPC systems were primarily at the first layers of the SC, whereas DPPC systems were more widely distributed. Systems with SCHOL had enhanced distribution and penetration of bicellar systems throughout the SC.

\section{Introduction}

Bicelles are described as discoidal nanostructures approximately $15-50 \mathrm{~nm}$ in diameter in which long-chain phospholipids, typically dimyristoyl-phosphatidylcholine or dipalmitoyl-phosphatidylcholine (DMPC or DPPC), form a flat bilayer and short-chain phospholipids, typically dihexanoyl-phosphatidylcholine (DHPC), stabilise the structure rim. ${ }^{1,2}$ These systems have been used for skin studies because bicelles do not require potentially irritant surfactants. ${ }^{3,4}$ Additionally, the nanostructures have a modular

\footnotetext{
${ }^{a}$ Department of Chemical and Surfactant Technology, Institute of Advanced Chemistry of Catalonia (IQAC-CSIC) C/Jordi Girona 18-26, 08034 Barcelona, Spain.E-mail: gelen.rodriguez@cid.csic.es; Fax: + 34-93 20459 04; Tel: + 34-934006161

${ }^{b}$ Synchrotron SOLEIL, SMIS Beamline, Gif-sur-Yvette, France

${ }^{c}$ University of Barcelona's Scientific and Technological Centers (CCiT-UB), Barcelona Science Park, C/Baldiri Reixac, 10, 08028 Barcelona, Spain
}

morphology and are small enough to penetrate the skin. ${ }^{4}$ Thus, these aggregates have potential dermatological, cosmetic, and pharmaceutical applications. Depending on the temperature, composition, and total lipid concentration, these nanostructures display a progression of morphologies from small discoidal micelles to cylindrical micelles and perforated lamellar sheets or vesicles. ${ }^{5,6}$ The remarkably versatile morphology of these systems is their most important characteristic, which facilitates their use in several fields. The effects of these systems on skin or, more specifically, the molecular organisation of stratum corneum (SC) lipids is a research focus of our group. ${ }^{4,7} \mathrm{The} \mathrm{SC}$ is a thin layer of flat anucleated cells (the corneocytes) surrounded by a lipid matrix and organised into bilayers (the intercellular lipids), which are a mixture comprising primarily ceramides, cholesterol and fatty acids as well as low levels of other lipids, such as cholesterol esters and cholesterol sulphate (SCHOL). The organisation and composition of SC lipids are responsible for skin barrier function. 
Despite the low proportion of SCHOL in skin (less than $1 \%$ of the total lipids), this lipid plays an important role in keratinocyte differentiation and development of the skin barrier. For example, a reduction of SCHOL content in the SC contributes to a delayed desquamation process, decreases the stability of bilayers and reduces the fraction of lipids arranged in liquid lateral packing. ${ }^{8}$ In addition, SCHOL can alter the physical properties of membrane models, which contributes to stable biological membranes and inhibits membrane fusion. ${ }^{8}$ SCHOL interacts with phospholipids in a manner similar to cholesterol and can induce changes in lipid bilayer ordering by forming a lamellar phase, the "liquid-ordered phase", with intermediate characteristics between solid ordered (gel) and liquid disordered phases. ${ }^{8,9}$

The topical use of classical bicelles is an advantageous strategy in skin applications, as our previous works have demonstrated. ${ }^{47}$ Aiming to make these systems more compatible with skin, this work proposes the use of SCHOL in forming new bicellar systems for applications in dermatology. First, the structures of the new systems that contain SCHOL instead of DHPC were characterised using an array of biophysical techniques, such as differential scanning calorimetry, fluorescence spectroscopy, X-ray scattering and freeze-fracture electron microscopy. This strategy supports a complete study on the thermotropic behaviour of these systems, which is important for understanding their effect on skin lipid organisation. ${ }^{7,10}$ Second, a comparative study of skin treated with SCHOL (DMPC/SCHOL and DPPC/ SCHOL) and DHPC systems (DMPC/DHPC and DPPC/DHPC) was performed. These experiments were conducted to determine how deep these systems penetrate the skin. In these studies, attenuated total reflectance-Fourier transform infrared (ATRFTIR) spectroscopy was used. The effect of different systems (with DHPC or SCHOL) on skin lipid phase behaviour was also studied using ATR-FTIR because the characteristic vibrational frequencies of the alkyl chain lipids are related to a different ordered phase. ${ }^{11}$ Furthermore, LAURDAN generalised polarisation (GP) images were acquired using two-photon excitation fluorescence (TPE) microscopy. This technique facilitated direct visualisation and measurements on lipid packing in SC lipid membranes. ${ }^{12}$ These complementary techniques may support a careful study on the effect of the different systems on SC lipid organisation.

\section{Materials and methods}

\section{Chemicals}

The 1,2-dimyristoyl-sn-glycero-3-phosphocholine (DMPC); 1,2-dipalmitoyl-sn-glycero-3-phosphocholine (DPPC); 1,2-dihexanoyl-snglycero-3-phosphocholine (DHPC); 1,2-dimyristoyl( $\left.\mathrm{d}_{54}\right)$-sn-glycero3-phosphocholine (DMPC- $\mathrm{d}_{54}$ ); 1,2-dipalmitoyl $\left(\mathrm{d}_{62}\right)$-sn-glycero-3phosphocholine (DPPC- $\mathrm{d}_{62}$ ) and 1,2-dihexanoyl $\left(\mathrm{d}_{22}\right)$-sn-glycero-3phosphocholine (DHPC- $\mathrm{d}_{22}$ ) were purchased from Avanti Polar Lipids (Alabaster, USA). The cholesteryl sulphate sodium salt (SCHOL) was acquired from Sigma-Aldrich GmbH (Steinheim, Germany). The chloroform and ethanol were purchased from Merck (Darmstadt, Germany). The purified water was generated through an ultra-pure water system, Milli-Q plus 185 (Millipore, Bedford, USA). The 6-lauroyl-2-( $N, N$-dimethylamino) naphthalene (LAURDAN) was purchased from Invitrogen, DK. The sodium dodecyl sulphate (SDS) solution was purchased from Sigma-Aldrich Chemie GmbH (Steinheim, Germany). Tape-stripping was performed using adhesive tape-stripping (D-squame ${ }^{\mathbb{R}}, \mathrm{CuDem}$ Inc., Dallas, USA).

\section{Preparation of the lipid systems}

Four binary lipid mixtures combining long-chain phospholipids (DMPC or DPPC) and DHPC or SCHOL were formed at a 3.5 : 1 lipid molar ratio. The systems formed with DHPC were mixed in chloroform, and those formed with SCHOL were mixed in chloroform-methanol $(2: 1 \mathrm{v} / \mathrm{v})$. The solvents were removed using a rotary evaporator, and the samples were kept under vacuum overnight. After this procedure, the systems were hydrated and subjected to several cycles of sonication and freezing until the samples were transparent. Air bubbles in the SCHOL samples were removed by centrifugation in an Eppendorf centrifuge 5415 (Hamburg, Germany) for $10 \mathrm{~min}$ at $14000 \mathrm{rpm}$. To generate the homogenised and stabilised samples, the SCHOL mixtures were kept several days at $-4{ }^{\circ} \mathrm{C}$. The same procedure was used to prepare the system formed with perdeuterated phospholipids for IR experiments.

\section{Differential scanning calorimetry (DSC)}

Calorimetric measurements for the DMPC/SCHOL and DPPC/SCHOL systems were performed using a DSC 821E Mettler Toledo (Greifensee, Switzerland) calorimeter. Hermetically sealed aluminium pans ( $40 \mu \mathrm{L}$ nominal volume) were used. Approximately $4 \mathrm{mg}$ of each lipid mixture was loaded into an aluminium pan and subjected to three heating-cooling cycles in the temperature range between -60 and $60{ }^{\circ} \mathrm{C}$ at a scanning rate of $5{ }^{\circ} \mathrm{C} \mathrm{min}^{-1}$. The data from the first scan were discarded to avoid mixing artefacts. The thermal cycles were repeated three times to ensure constancy and reproducibility of the data as well as to erase the thermal history of the sample. Due to supercooling, only heating scans were used in this work. ${ }^{13}$ The temperature at the peak maximum that corresponded to thermotropic transitions was defined as the transition temperature. Enthalpies were calculated by integrating the peak area after baseline adjustment and normalising to the sample quantity analysed. DSC curves were generated and analysed by STAR 9.2 Software provided by Mettler Toledo.

\section{LAURDAN generalised polarisation (GP) measurements}

LAURDAN in ethanol was added to the previously prepared systems using a $300: 0.79$ lipid-probe ratio (the final concentration of LAURDAN was $1.5 \mu \mathrm{M}$ ). The emission spectra were collected in the range of temperatures from $15{ }^{\circ} \mathrm{C}$ to $50{ }^{\circ} \mathrm{C}$. The samples were prepared and stored in dark, and the emission spectra were measured $24 \mathrm{~h}$ after preparation.

The LAURDAN generalised polarisation (GP) denotes the position of the probe's emission spectra. ${ }^{14}$ The fluorescence emission properties of LAURDAN are sensitive to the water dipolar relaxation process in the probe's local environment (the lipid bilayer in this case). The extent of water dipolar relaxation observed in the gel phase is low compared with relaxation in the liquid disordered phase. ${ }^{14}$ As a consequence, the probe's emission is blue in the gel phase 
and green in the liquid disordered phase. ${ }^{14}$ The LAURDAN GP provides information on lipid bilayer lateral packing. ${ }^{14}$ The GP function is analogous to the fluorescence polarisation, as follows:

$$
\mathrm{GP}=\left(I_{\mathrm{B}}-I_{\mathrm{R}}\right) / I_{\mathrm{B}}+I_{\mathrm{R}}
$$

where $I_{\mathrm{B}}$ and $I_{\mathrm{R}}$ correspond to intensity at the blue and red regions of the emission spectrum, respectively, using a given excitation wavelength. ${ }^{14}$ A Chronos ISS fluorometer (ISS, Champaign, IL) was used to measure GP in the LAURDANlabelled systems. The excitation wavelength was $374 \mathrm{~nm}$ (using a laser diode as the excitation source). The emission wavelengths were $440 \mathrm{~nm}\left(I_{\mathrm{B}}\right)$ and $490 \mathrm{~nm}\left(I_{\mathrm{R}}\right)$; see eqn (1). The LAURDAN GP values are plotted as a function of temperature, and the inflection point of the curve is the transition temperature of the system.

\section{X-ray scattering: small- (SAXS) and wide-angle (WAXS)}

$\mathrm{X}$-ray scattering at small (SAXS) and wide angles (WAXS) was performed using a S3-MICRO (Hecus X-ray systems GMBH Graz, Austria) coupled to a GENIX-Fox 3D X-ray source (Xenocs, Grenoble), which produces a detectorfocussed X-ray beam with $\lambda=0.1542 \mathrm{~nm} \mathrm{Cu} \mathrm{K} \alpha$-line at greater than $97 \%$ purity and less than $0.3 \% \mathrm{~K} \alpha$. The transmitted scattering was detected using two PSD 50 Hecus, one in the small-angle regime $\left(0.09 \mathrm{~nm}^{-1}<q<6 \mathrm{~nm}^{-1}\right)$ and another in the wide-angle regime $\left(13 \mathrm{~nm}^{-1}<q<19 \mathrm{~nm}^{-1}\right)$. Temperature was controlled through a Peltier TCCS-3 Hecus. The samples were inserted into a flow-through glass capillary with a diameter of $1 \mathrm{~mm}$ and a wall thickness of $20 \mu \mathrm{m}$. The SAXS scattering curves are plotted as a function of the scattering vector modulus,

$$
q=\frac{4 \pi}{\lambda} \times \sin \left(\frac{\theta}{2}\right)
$$

where $\theta$ is the scattering angle. The $q$ values in our system ranged from $0.08 \mathrm{~nm}^{-1}$ to $6.0 \mathrm{~nm}^{-1}$ in the SAXS regime and from 12 to $20 \mathrm{~nm}^{-1}$ in the WAXS regime. The system scattering vector was calibrated by measuring a standard silver behenate sample. The scattering curves were primarily smeared by the detector width because we used a detectorfocussed small beam $(300 \times 400 \mu \mathrm{m}$ full width at half maximum), which widens the peaks without a noticeable effect on the peak position. The background was subtracted from the scattering curves. These curves were placed on an absolute scale by comparison with water scattering. ${ }^{15,16}$ The instrumentally smeared experimental SAXS curves were fit to numerically smeared models for beam size and detector width effects. A least squares routine based on the Levenberg-Marquardt scheme was used. ${ }^{17}$ The bilayer was fit using a three-Gaussian profile based on the MCG model used by Pabst et al. ${ }^{18}$ To improve the bilayer description, a methylene electronic density step was added to the bilayer hydrophobic region with a width that corresponded to \pm headgroup-headgroup distance $\left(Z_{\mathrm{H}}\right)$, and the medium contrast was set to the electronic density of water. The remaining parameters and the model can be found in Pabst et al. ${ }^{18}$

\section{Freeze-fracture electron microscopy (FFEM)}

FFEM is a technique that involves cryofixation, replication and transmission electron microscopy. This technique is widely used to characterise lipid bilayer and non-bilayer arrangements ${ }^{19}$ as well as monolayer-based structures. ${ }^{20}$ FFEM avoids artefacts from composition changes are inherent in chemical fixation, staining and drying techniques. The procedure performed for the FFEM study herein was based on the study described by Egelhaaf et al. ${ }^{21}$ Freezefracture samples were prepared by depositing approximately $1 \mu \mathrm{L}$ of the sample between two copper platelets using a 400-mesh gold grid as a spacer. The samples were then frozen by plunging into a liquid propane bath cooled to $-180{ }^{\circ} \mathrm{C}$ by liquid nitrogen and fractured by separating the two copper plates in a BalTec BAF 300 freeze-etching device (Leica, Vienna, Austria) at $-110{ }^{\circ} \mathrm{C}$ and $5 \times$ $10^{-8}$ mbar. Replicas were generated through unidirectional shadowing at $45^{\circ}$ with $2 \mathrm{~nm}$ of platinum/carbon $(\mathrm{Pt} / \mathrm{C})$ and at $90^{\circ}$ with $20 \mathrm{~nm}$ of carbon. After replication, the samples were brought to room conditions. An advantage of this preparation design is that large portions of the metal replicas were automatically attached to the TEM grids. The grids and replicas were cleaned by immersion in concentrated sodium hypochlorite solution to completely remove any organic material from the sample and leave only the $\mathrm{Pt} / \mathrm{C}$ replica. The replicas were examined in a Jeol 1010 Transmission electron microscope (Jeol, Tokyo, Japan) at $80 \mathrm{kV}$.

\section{Skin preparation and treatment with bicellar systems}

Porcine skin was obtained from the backs of experimental animals in the Faculty of Veterinary, Universitat Autònoma de Barcelona and Department of Dermatology, Hospital Clinic of Barcelona (Spain) 2-3 hours after the animals were sacrificed. The bristles were removed carefully using an animal clipper, and the skin then was washed with tap water. The excised skin was dermatomed to $500 \pm 50 \mu \mathrm{m}$ thicknesses (Dermatome GA630, Aesculap, Tuttlingen, Germany).

Four bicellar systems were applied to the skin: DMPC/ DHPC, DPPC/DHPC, DMPC/SCHOL and DPPC/SCHOL. For the IR experiments, the bicellar systems contained the following perdeuterated phospholipids: DMPC- $\mathrm{d}_{54} / \mathrm{DHPC}_{-\mathrm{d}}$; DPPC-d $\mathrm{d}_{62} /$ DHPC- $\mathrm{d}_{22} ;$ DMPC- $\mathrm{d}_{54} /$ SCHOL and DPPC- $\mathrm{d}_{62} /$ SCHOL. An additional method, tape stripping, was used in combination with ATR-FTIR spectroscopy to determine the localisation and the effect of the systems at different SC depths. This procedure consisted of removing the SC layers through sequential application and removal of adhesive tapes. The stripping technique began several days after treatment with the bicellar systems in SC was completed. Each strip was applied to the skin at a constant pressure $\left(86 \mathrm{~g} \mathrm{~cm}^{-2}\right)$ for 10 s. For each repeated strip, the tearing-off direction was varied by $90^{\circ}$ for homogeneous removal of the SC cell layers. Due to this standardised procedure, each cell layer removed had nearly the same thickness, ${ }^{22}$ to increase reproducibility, the procedure was performed by the same person in accordance with the AAPS/FDA Workshop Report ${ }^{23}$ and Guidance for Industry. ${ }^{22}$ ATR-FTIR spectroscopy measurements were performed after each strip. For the TPE microscopy experiments, before treatment with bicellar systems, the skin was incubated overnight with $5 \mu$ of the LAURDAN stock solution in dimethyl sulphoxide (DMSO) at a final concentration of $100 \mu \mathrm{M}$. 
The skin pieces were treated with the SC side up over a thin layer of water (but not submerged) on a Petri dish at $37^{\circ} \mathrm{C}$, which was controlled using a thermostat. Under these specific conditions, complete hydration of the skin samples was maintained. Treatment with bicellar systems or water (the control sample) consisted of four applications (every $1 \mathrm{~h}$ ) of $1 \mathrm{mg}$ lipid $\mathrm{cm}^{-2}$ of the bicellar system or water on the skin surface. At the end of treatment (4 applications), the samples were washed with an SDS solution (at $0.5 \% \mathrm{w} / \mathrm{v}$ ) and then with Milli-Q water.

\section{IR experiments}

IR measurements were performed at the SMIS beamline of the Synchrotron SOLEIL. A Thermo Continuum XL IR microscope coupled to an FTIR Nicolet Nexus 5700 spectrometer was employed in these experiments. The system comprises a mercury-cadmium-telluride (MCT) type A detector cooled by liquid nitrogen and an $X-Y-Z$ motorised stage with $1 \mu \mathrm{m}$ incremental steps. For this study, a micro-slide-on the ATR silicon crystal (refractive index $n=3.4$ ) was directly connected to the objective with an aperture of $150 \mu \mathrm{m} \times 150 \mu \mathrm{m}$ that generated investigation areas of approximately $40 \mu \mathrm{m} \times 40 \mu \mathrm{m}$.

Optical micrographs were produced from the microscope in the visible image mode to define the sampling positions, and IR spectra were collected by gently lowering the ATR crystal onto the sample surface and applying pressure until optimum contact. The spectra were acquired at room temperature in the $4000-650 \mathrm{~cm}^{-1}$ range and a spectral resolution of $4 \mathrm{~cm}^{-1}$, and 128 scans were averaged. To analyse the original spectra, a fitting procedure, which included a number of individual Gaussian peaks, was calculated to fit a complex set of overlapping peaks in the spectrum. A Savitsky-Golay second derivative procedure was used to locate peak positions using the Nicolet Omnic-Atlus software. The measurements were performed in triplicate, and the statistical analyses were performed using Dixon's test to detect outliers, which were excluded from the data. One-way analysis of variance and the Fisher test were used to determine significant differences between the values obtained from the different treatments with the control sample (significance level accepted, ${ }^{*} P<0.05$ ).

\section{Two-photon excitation fluorescence microscopy experiments}

The measurements were performed using a custom-built multiphoton excitation system constructed in a Nikon Eclipse TI microscope. The objective was a $40 \times$ water immersion objective, NA 1.20. The excitation light source was a femtosecond Ti:Sa laser (HPe Mai Tai DeepSee, tunable excitation range 690-1040 nm, Spectra Physics, Mountain View, CA), and the excitation wavelength was $780 \mathrm{~nm}$. The fluorescent signals from the probe's emission spectrum were split using a dichroic mirror centred at $470 \mathrm{~nm}$ and collected using band-pass filters at $438 \pm$ $12 \mathrm{~nm}$ and $494 \pm 10 \mathrm{~nm}$ simultaneously using two Hamamatsu H7422P-40 photomultipliers. Based on the intensity images, the LAURDAN GP images were computed according to eqn (1) using SimFCS software (Laboratory for Fluorescence Dynamics, Irvine, CA). The GP images were calibrated with correction factor G generated from a LAURDAN GP standard ( $2 \mu$ M LAURDAN in DMSO) as previously described. ${ }^{24}$ The LAURDAN GP value for the reference solution was measured in an ISS
Chronos spectrofluorometer (ISS, Champaign, USA), as described above. The pixel distribution of the GP values is shown as a GP histogram generated from a region of interest in the image, and the average GP value is from the centre of the GP histogram (see Results section). High LAURDAN GP values ( $>0.6)$ correspond to a solid ordered (gel) phase, whereas low LAURDAN GP values $(<-0.2)$ correspond to liquid disordered phases. ${ }^{12}$ For microscope observations, the skin was mounted on a glass-bottom dish with the dermal side contacting buffer-soaked filter paper to avoid dehydration. Images of the pig skin samples were taken from the SC surface toward the inside. The statistical analyses were performed using Dixon's test to detect outliers, which were excluded from the data.

\section{Results}

\section{Characterisation of the bicellar systems}

DSC and bulk LAURDAN GP experiments. The DSC technique facilitates thermotropic behaviour studies on the systems formed by SCHOL and long-chain phospholipids (DPPC or DMPC). Both cooling and heating curves were determined. The enthalpy values were within the expected experimental errors, and the cooling calorimetric plots corroborated the heating results. Thus, herein, we only considered the heating curves.

Fig. 1A shows the DSC data generated from the DPPC/ SCHOL system (black line graph). This sample displays an endothermic sharp peak centred at $34.3{ }^{\circ} \mathrm{C}$ with an enthalpy variation of $18.8 \mathrm{~kJ} \mathrm{~mol}^{-1}$. This sharp peak corresponds to a phase transition with high cooperativity. For pure DPPC, a symmetric sharp peak is reported at a higher temperature, $41{ }^{\circ} \mathrm{C}$, which corresponds to the main transition from the gel to the liquid disordered phase. ${ }^{25}$ The high symmetry indicates a single phase $;{ }^{26}$ in contrast, the peak is slightly asymmetrical for the DPPC/SCHOL system, which corresponds to more than one phase. ${ }^{27}$

Fig. 1A further shows the LAURDAN GP temperature profile for the DPPC/SCHOL system (blue line). The LAURDAN GP curve has an inflection point at approximately $34{ }^{\circ} \mathrm{C}$, which indicates a substantial change in the level of water dipolar relaxation around the probe. This inflection point can be assigned to a lipid phase transition similar to the pure DPPC transition from a gel to a liquid disordered phase. However, the lower GP values are higher, and the transition cooperativity (indicated by the slope) is slightly lower compared with pure DPPC. This result correlates with the melting phase transition temperature observed in the DSC experiment. Thus, both techniques indicate a main transition temperature $\left(T_{\mathrm{m}}\right)$ from a gel to a liquid disordered organisation.

The thermotropic behaviour of the DMPC/SCHOL sample is represented in Fig. 1B. The black line graph represents the DSC curve for the temperature range studied; no peaks associated with a main lipid transition are observed in this range; the peak at approximately $5{ }^{\circ} \mathrm{C}$ is from ice melting. ${ }^{28} \mathrm{To}$ confirm that the peak observed at approximately $5{ }^{\circ} \mathrm{C}$ is from ice melting and to discard contributions from DMPC/ SCHOL, a DSC curve was also generated from $0{ }^{\circ} \mathrm{C}$ to $60{ }^{\circ} \mathrm{C}$ (data not shown). Thus, the ice does not interfere with the measurements, and no peaks were observed. The inset shows 

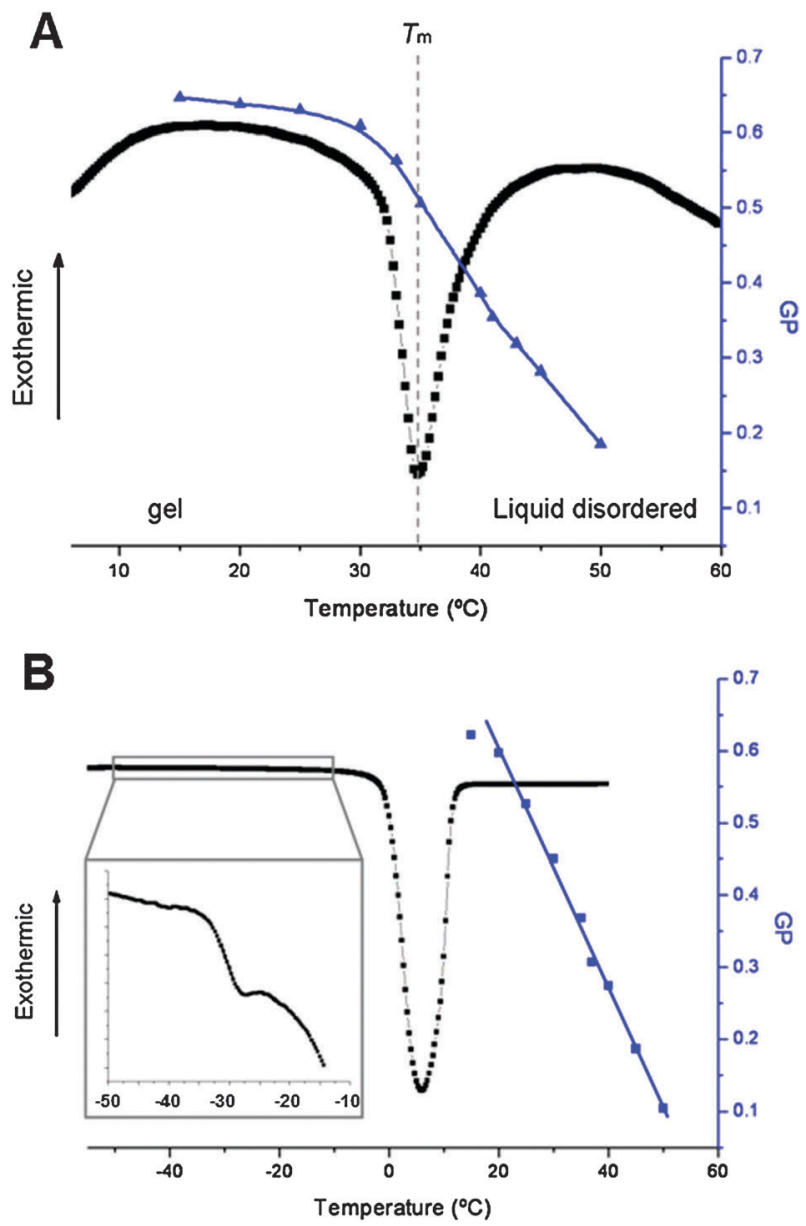

Fig. 1 DSC experiments (black line) and the LAURDAN GP temperature profile (blue line) for the DPPC/SCHOL (A) and DMPC/SCHOL systems (B). The inset in panel B shows the DSC curve enlargement from $-50{ }^{\circ} \mathrm{C}$ to $-10{ }^{\circ} \mathrm{C}$.

the DSC analysis between $-50{ }^{\circ} \mathrm{C}$ and $-10{ }^{\circ} \mathrm{C}$. A small thermotropic change was observed at $-28.3{ }^{\circ} \mathrm{C}$ with an enthalpy variation of $2.5 \mathrm{~kJ} \mathrm{~mol}^{-1}$. This transition shows an asymmetric and broad shoulder, which indicates a weak interaction between molecules. Thus, this transition should not correspond with the expected $T_{\mathrm{m}}$ for pure DMPC from a gel to the liquid disordered phase that has been reported at $23{ }^{\circ} \mathrm{C}^{25}$ The LAURDAN GP temperature profile was also observed (blue line) with no inflection at this temperature range. Although LAURDAN experiments could lead to an inflection below $15{ }^{\circ} \mathrm{C}$, which denotes a phase transition, the DSC results did not show any change at this temperature range $\left(-60\right.$ to $\left.60{ }^{\circ} \mathrm{C}\right)$; thus, we assume that the main transition seems to be suppressed in the system studied. This different SCHOL effect on both phospholipids (DMPC and DPPC) is surprising. For DPPC membranes, the $T_{\mathrm{m}}$ is decreased a few degrees, while for the DMPC membranes, $T_{\mathrm{m}}$ is suppressed. However, the SCHOL concentration is the same for both systems (23 mol\%). This point is explained in the Discussion section.

\section{X-ray scattering}

Small angle $X$-ray scattering ( $S A X S)$. SAXS provides information on the large structural features of the sample, primarily the bilayer characteristics, and repeat distance ( $d$-spacing) for the lamellar phase described by Bragg's law. Panels A and B (in Fig. 2) show the best fit (solid line) for the original pattern (open circles) in DPPC/SCHOL and DMPC/ SCHOL systems, respectively, at room temperature. The fit of the MCG model to the experimental points is good over the entire $q$ range except for the points at approximately $q=$ $0.4 \mathrm{~nm}^{-1}$; this could be a consequence of certain features that are not captured by the model. Moreover, the electron density profile in the direction perpendicular to the membrane is represented in the insets of these panels. The structural parameters generated from the fits are similar for both systems and are listed in Table 1. The fits are produced for the lamellar structures at a repetition distance $(d)$ of $52.50 \mathrm{~nm}$ for DPPC/ SCHOL and $37.1 \mathrm{~nm}$ for the DMPC/SCHOL system with an average of 5 correlated bilayers $(N)$. In both cases, the first and second reflections are observed as bumps or inflections, albeit with strong instrumental smearing due to the large distances involved. Moreover, the Caille parameter $(\eta)$, which is related to membrane elasticity, has similar values (0.10 and 0.09$)$ for DPPC/SCHOL and DMPC/SCHOL, respectively. In contrast, the $\eta$ for pure DPPC [R. Pons unpublished results] and $\mathrm{DMPC}^{29}$ is smaller (0.008 and 0.077, respectively), which indicates higher flexibility for membranes with SCHOL. The bilayer thickness $\left(d_{\mathrm{B}}\right)$ was directly determined using the following equation:

$$
d_{\mathrm{B}}=2\left(Z_{\mathrm{H}}+2 \sigma_{\mathrm{H}}\right)
$$

where $Z_{\mathrm{H}}$ and $\sigma_{\mathrm{H}}$ are the centre and width, respectively, for the headgroup Gaussians. ${ }^{18}$ The calculated $d_{\mathrm{B}}$ is 6.02 for the DPPC/ SCHOL system and $5.12 \mathrm{~nm}$ for DMPC/SCHOL. Differences between the structural parameters are discussed below.

Wide angle $X$-ray scattering (WAXS). WAXS provides information about the scattered intensity at wider angles than SAXS. Thus, we can acquire information on the smaller structural units in the sample, such as lateral packing in the lamellar phase. The C and D panels in Fig. 3 show WAXS profiles for DPPC/SCHOL and DMPC/SCHOL, respectively. In both cases, only one reflection was observed $\left(14.8 \mathrm{~nm}^{-1}\right.$ and at $14.5 \mathrm{~nm}^{-1}$ for DPPC/SCHOL and DMPC/SCHOL, respectively). This reflection corresponds to Bragg distances at approximately $0.42 \mathrm{~nm}$, which are more visible in the DPPC/ SCHOL sample than the DMPC/SCHOL sample. This distance is associated with a gel organisation of the systems specifically in the hexagonal phase. ${ }^{30}$

Freeze-fracture electron microscopy (FFEM). This technique is useful for observing the structural morphologies and topologies in these systems. Fig. 3 shows the most representative micrographs of the systems, DMPC/SCHOL (right panels) and DPPC/ SCHOL (left panels). Both samples show large lamellar stacks (Fig. 3A and B) and small structures at approximately $40 \mathrm{~nm}$ for DMPC/SCHOL (Fig. 3C) and 100-40 nm for DPPC/SCHOL (Fig. 3D). In addition, Fig. 3E and $\mathrm{F}$ show a long ripple phase at $25 \mathrm{~nm}$.

This structural variety contrasts with the morphologies for the DHPC systems reported in previous works by our group. ${ }^{31,32}$ In those results, only small discoids without stacked structures were 

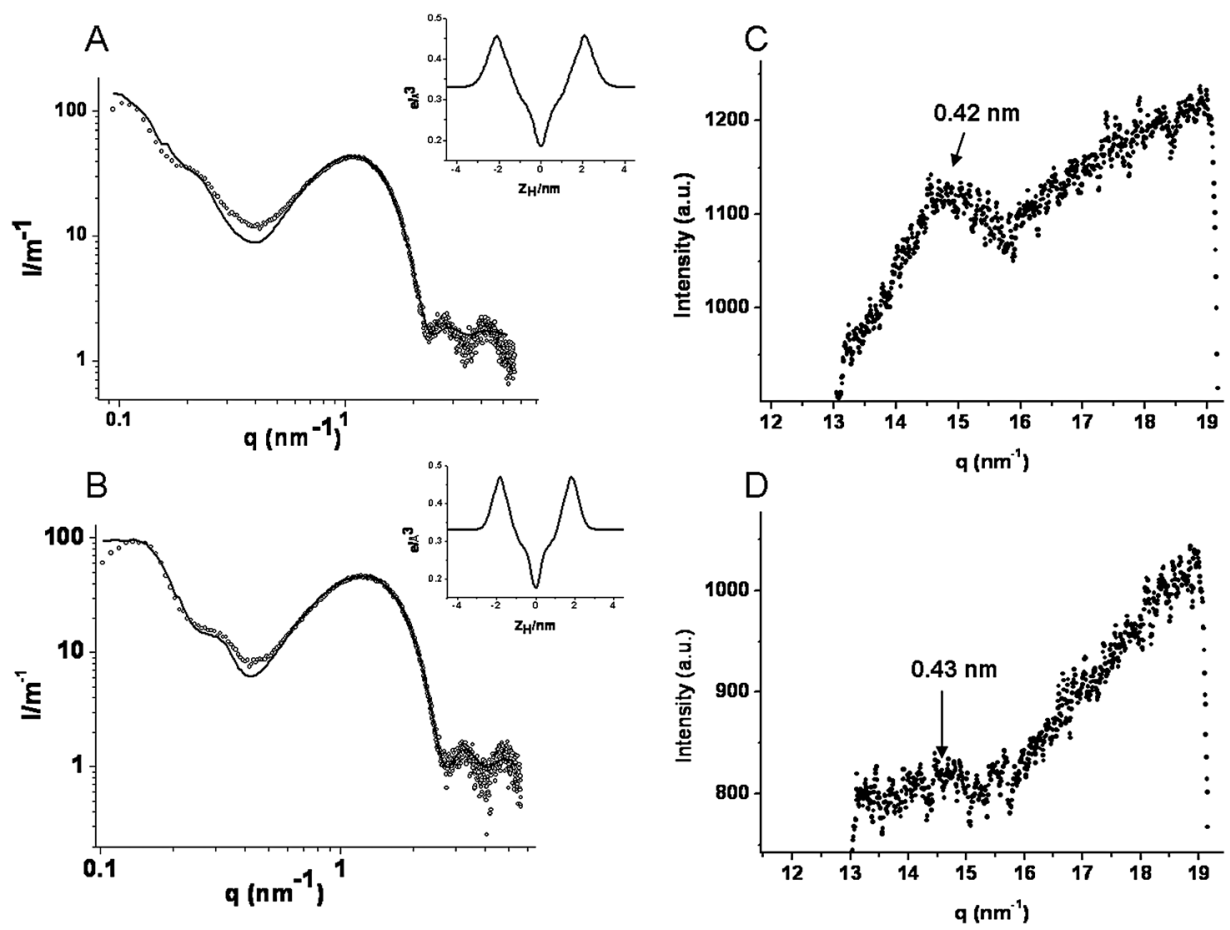

Fig. 2 Small- (left panels) and wide-angle (right panels) X-ray scattering intensity patterns recorded at room temperature for the DPPC/SCHOL (A and C panels) and DMPC/SCHOL systems (B and D panels). The insets show the electron density profiles for the membranes.

Table 1 Fit results for the scattering patterns of DPPC/SCHOL and DMPC/SCHOL systems

\begin{tabular}{|c|c|c|}
\hline Parameters & DPPC/SCHOL & DMPC/SCHOL \\
\hline$D$ & $52.50 \pm 3 \mathrm{~nm}$ & $37.10 \pm 1 \mathrm{~nm}$ \\
\hline$d_{\mathrm{B}}$ & $6.02 \pm 0.05 \mathrm{~nm}$ & $5.12 \pm 0.05 \mathrm{~nm}$ \\
\hline$d_{\mathrm{W}}$ & $46.48 \pm 3 \mathrm{~nm}$ & $31.48 \pm 1 \mathrm{~nm}$ \\
\hline$\eta$ & $0.10 \pm 0.01$ & $0.09 \pm 0.01 \mathrm{~nm}$ \\
\hline$N$ & $5 \pm 1$ & $5 \pm 1$ \\
\hline$\sigma_{\mathrm{H}}$ & $0.54 \pm 0.05$ & $0.43 \pm 0.05$ \\
\hline$\sigma_{\mathrm{C}}$ & $0.28 \pm 0.02$ & $0.22 \pm 0.02$ \\
\hline$\rho_{\mathrm{H}}$ & $0.47 \pm 0.02{\mathrm{e} \AA^{-3}}^{-3}$ & $0.48 \pm 0.02{\mathrm{e} \AA^{-3}}^{-3}$ \\
\hline$\rho_{\mathrm{C}}$ & $0.24 \pm 0.02 \mathrm{e}^{-3}$ & $0.23 \pm 0.02 \mathrm{e}^{-3}$ \\
\hline$Z_{\mathrm{H}}$ & $1.93 \pm 0.02 \mathrm{~nm}$ & $1.71 \pm 0.02 \mathrm{~nm}$ \\
\hline
\end{tabular}

$d$ Bragg distance; $d_{\mathrm{B}}$ bilayer thickness; $d_{\mathrm{W}}$ water layer thickness; $\eta$ Caille parameter; $N$ number of correlated bilayers; $\sigma_{\mathrm{H}}$ and $\rho_{\mathrm{H}}$ headgroup width and electron density; $\sigma_{\mathrm{C}}$ and $\rho_{\mathrm{C}}$ methyl width and electron density; and $Z_{\mathrm{H}}$, headgroup-headgroup distance. The meaning of the different parameters is as in Pabst et al. ${ }^{18}$

visualised. Thus, the microscopy evidences the noteworthy effect of SCHOL on assembly of lipid aggregates.

\section{Skin analysis}

FTIR experiments. Attenuated total reflectance-Fourier transform infrared spectroscopy (ATR-FTIR) is a technique in which the sample is placed directly on an ATR crystal. The penetration depth of IR in the skin is approximately $1 \mu \mathrm{m} .{ }^{33}$ Thus, this is a useful technique for investigating the SC without isolation from the other skin layers. The following two types of information are generated by combining this technique with the tape stripping method: the distribution of the bicellar system through the SC and the effect on SC lipids through treatment with this bicellar system. The IR spectra of the skin show at
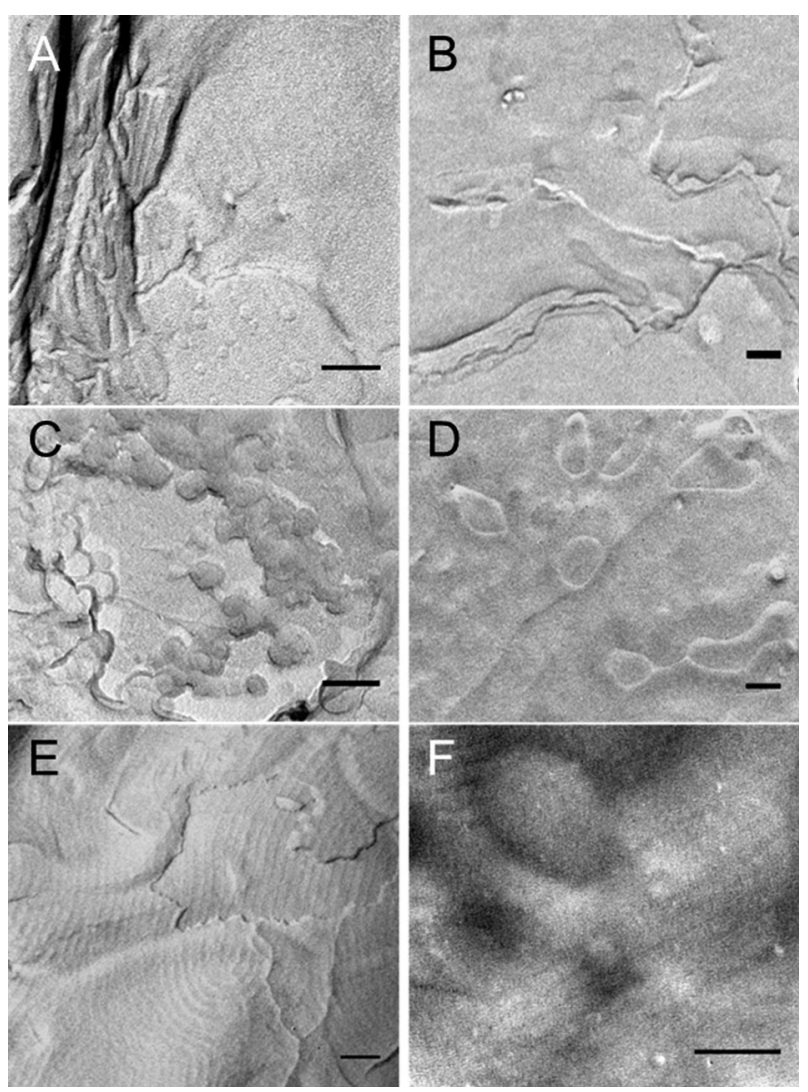

Fig. 3 The most representative micrographs from Freeze-Fracture Electron Microscopy of the SCHOL systems, DMPC/SCHOL (right panels) and DPPC/SCHOL (left panels). (A and B) Large lamellar stacks, (C and D) small structures, and (E and F) long ripple phase. Scale bar, $100 \mathrm{~nm}$. 
least three bands associated with SC polypeptides and proteins, as follows: amide A (NH vibration, approximately $3300 \mathrm{~cm}^{-1}$ ), amide $\mathrm{I}\left(\mathrm{C}=\mathrm{O}\right.$ vibration, approximately $\left.1650 \mathrm{~cm}^{-1}\right)$, and amide II $\left(\mathrm{C}-\mathrm{N}\right.$ vibration, approximately $\left.1550 \mathrm{~cm}^{-1}\right)$. Bands associated with the alkyl chains of $\mathrm{SC}$ lipids through the $\mathrm{CH}_{2}$ stretching vibration (approximately 2900 and $2850 \mathrm{~cm}^{-1}$ asymmetric and symmetric, respectively) are also visible; ${ }^{34}$ see Fig. 4A.

Distribution of the perdeuterated phospholipids. To study the distribution of the bicellar systems through the SC, the systems were produced using perdeuterated phospholipids. Perdeuterated systems for skin treatment in FTIR experiments have the advantage that the CD mode appears in a region of the spectra without a vibrational feature associated with the skin $\left(2000-2300 \mathrm{~cm}^{-1}\right.$ range, see Fig. 4A). This facilitates analysis of the different characteristic bands associated with the skin, such as for intrinsic lipids $\left(\mathrm{CH}_{2}\right.$ vibration in Fig. 4A) or skin proteins (amide I and amide II vibrations in Fig. 4A) and exogenous lipids (CD in Fig. 4A) without a band overlap. Fig. 4B shows a normalised absorbance for the $\mathrm{CD}$ vibration (system signal) with respect to the penetration depth in the skin. This parameter is calculated as the quotient between the areas under the curves (AUC) for the CD and the
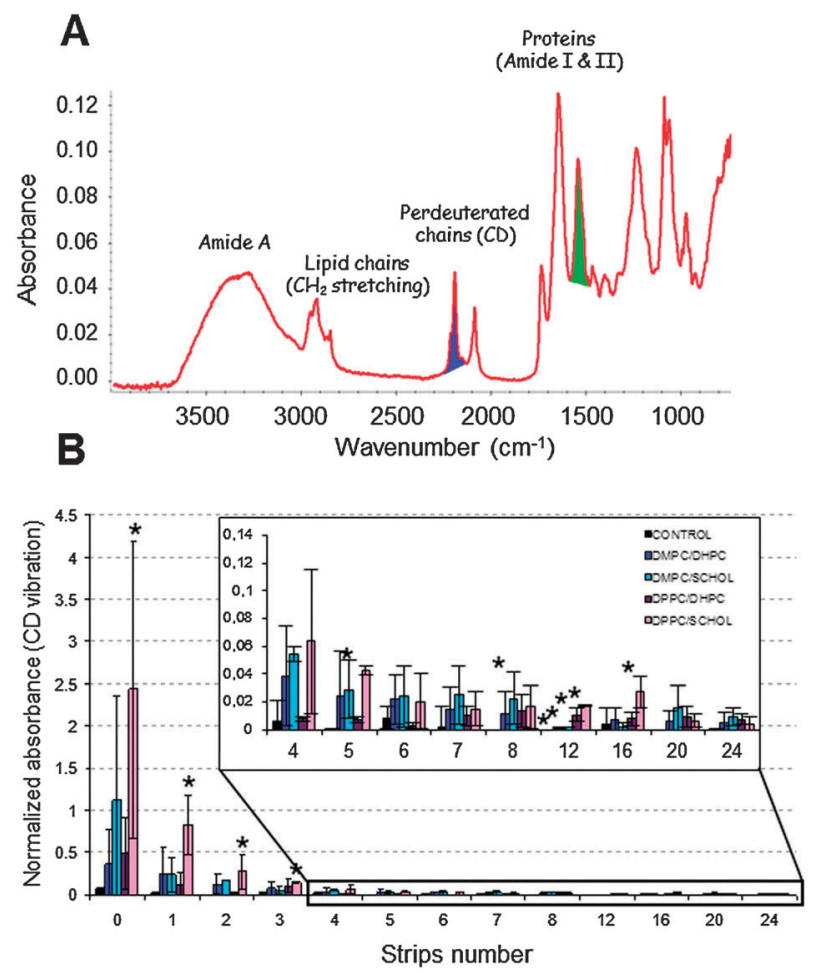

Fig. 4 Representative ATR-FTIR spectrum at room temperature for skin treated with the perdeuterated system. (A) Bands associated with skin vibration such as the alkyl chain of SC lipids $\left(\mathrm{CH}_{2}\right.$ stretching $)$ and $\mathrm{SC}$ proteins, including amide $\mathrm{A}(\mathrm{N}-\mathrm{H}$ vibration $)$, amide $\mathrm{I}(\mathrm{C}=\mathrm{O}$ vibration), and amide II (C-N vibration), and bands associated with exogenous vibration ( $\mathrm{CD}$ vibration) are indicated in panel $\mathrm{A}$. The area under curve (AUC) is from an internal reference (amide II) in green, and the AUC from an external reference (perdeuterated vibration) is in blue. They were selected to normalise the absorbance of the $\mathrm{CD}$ vibration, which is (B) plotted as a function of penetration depth through the skin for the different systems. The inset shows an enlarged fourth strip. The significance level accepted, ${ }^{*} P<0.05$. amide II vibrations (skin signal), $\mathrm{AUC}_{\mathrm{CD}} / \mathrm{AUC}_{\text {Amide II }}$, and it indicates the exogenous lipid quantities in the skin after application of each system. Generally for these systems, the levels of exogenous lipids are higher at superficial than deep layers of the skin. The systems were detected at high depths (see the inset in Fig. 4B), and significant differences compared with the control sample (see * in the inset, Fig. 4B) were noted at the 12th strip. Systems with SCHOL were significantly different at high depths; this effect is especially noted in the DPPC/SCHOL system at the 16th strip. The samples treated with DPPC systems follow a pattern where the distribution is more homogeneous into the skin than samples treated with DMPC systems because from the first strip the normalised absorbance values are nearly the same at all depths.

Effect of systems on SC lipids. To study how deep lipids from bicelles affect $\mathrm{SC}$ lipids, the $\mathrm{CH}_{2}$ stretching vibration that corresponds to SC lipids was analysed. This vibration provides information about the conformational order-disorder transitions from a gel (orthorhombic or hexagonal phase, 2849 or $2850 \mathrm{~cm}^{-1}$, respectively) to a liquid-crystalline phase $\left(2852 \mathrm{~cm}^{-1}\right) .{ }^{34}$ Fig. 5 shows the mean variations $(n=3)$ in the $\mathrm{CH}_{2}$ stretching vibration after stripping. The control sample shows a lower and constant wavenumber compared with treated samples at all depths. The most striking changes were observed for treated samples at 0 strips. The highest wavenumber was observed for the DMPC/ DHPC sample at $2852.48 \pm 0.3 \mathrm{~cm}^{-1}$, which indicates high levels of lipid disorganisation. In contrast, the samples treated with the DPPC/DHPC system had the lowest wavenumbers of the treated samples $\left(2849.6 \pm 0.5 \mathrm{~cm}^{-1}\right)$, which indicates a higher lipid organisation. The samples treated with the SCHOL systems had intermediate values between the DHPC systems (2850.80.8 $\mathrm{cm}^{-1}$ for DMPC/SCHOL and $2849.6 \pm 0.5 \mathrm{~cm}^{-1}$ for DPPC/SCHOL). Despite these differences, after the first strip the treated samples had a $\mathrm{CH}_{2}$ stretching value between 2849 and $2850 \mathrm{~cm}^{-1}$, which corresponds to an ordered lipid phase (gel phase).

Two-photon excitation fluorescence microscopy experiments. Fig. 6 shows the resulting image (GP image) of the skin surface from untreated (control) and DMPC/DHPC-treated systems. The GP image characteristics for SC comprise black areas (non-fluorescent) that correspond to corneocytes, and surrounding these regions are fluorescent areas associated with a lipid matrix because the probe (LAURDAN) is localised in the lipid bilayers. ${ }^{12}$ For the control sample, the average GP had high values (colour-coded red), which indicates highly organised packing in the SC lipid, while for skin treated with the DMPC/DHPC system, the GP values are lower (colour-coded as yellow-red), which corresponds to an increase in dipolar relaxation (water content and dynamics) related to disorganisation in the lamellar structure. Fig. 7 plots the difference in GP values between the control and treated samples $\left(\Delta \mathrm{GP}=\mathrm{GP}_{\text {control }}-\right.$ $\left.\mathrm{GP}_{\text {treated }}\right)$. The $\Delta \mathrm{GP}$ are positive values, and GP control $>\mathrm{GP}$ treated; as a consequence, the SC lipids are less organised after any of the treatments. The most significant GP difference is noted for skin treated with the DMPC/DHPC system at all the depths analysed. Thus, this system induced the strongest influence on SC lipid organisation. 


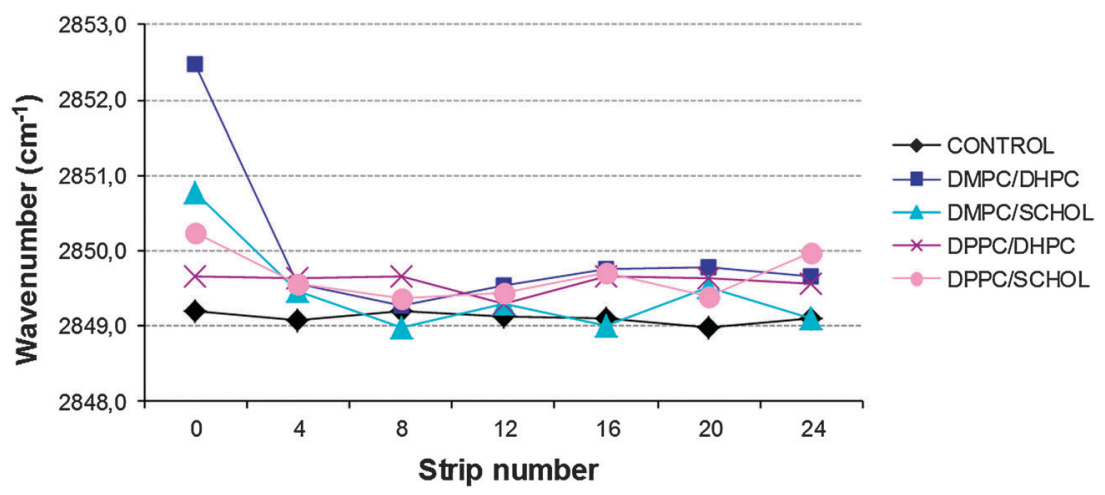

Fig. 5 The mean variation in the $\mathrm{CH}_{2}$ stretching vibration $(n=3)$ after tape strippings for the different systems. The standard deviations vary between 0.8 and 0.2 .

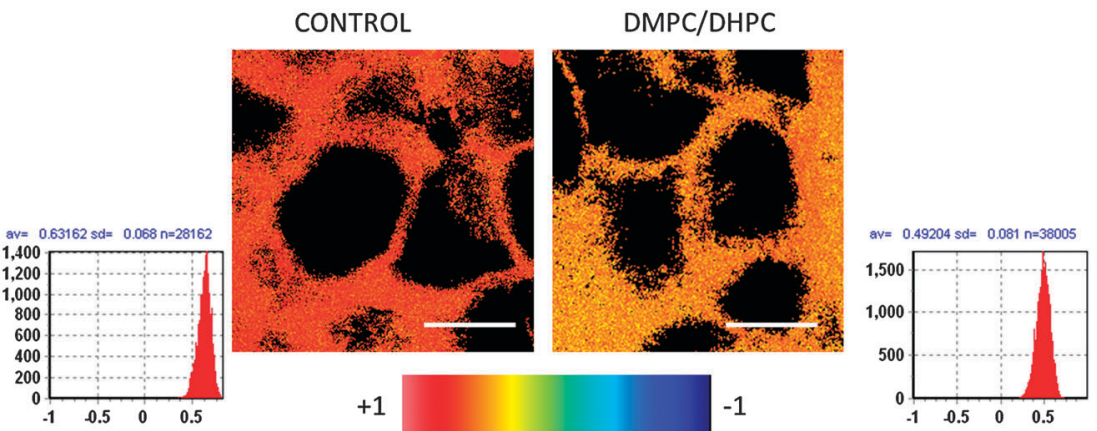

Fig. 6 LAURDAN GP images of the skin surface, untreated (control) and treated with the DMPC/DHPC system. The black areas (nonfluorescent) are the corneocytes, and the lipid matrix surrounds them (fluorescent areas). The histograms represent the average GP value for each image, and the colour scale is from +1 to -1 . Scale bar, $20 \mu \mathrm{m}$.

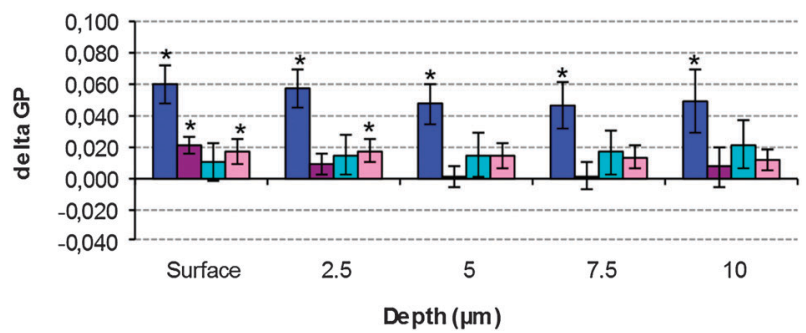

Fig. 7 The change in GP values $(\Delta \mathrm{GP})$ between the control and treated samples at different depths through the skin for the DMPC/ DHPC (the dark blue bar) and DMPC/SCHOL systems (the light blue bar), DPPC/DHPC (the violet bar) and DPPC/SCHOL systems (the pink bar) are plotted. The standard error of the mean was produced by dividing the standard deviation of the original distribution by the root square of sample size (sample size $>8$ ), and the significant level accepted was $* \Delta \mathrm{GP}>2 \mathrm{sd}$

\section{Discussion}

\section{Characterisation of the systems}

Characterisation of the typical DMPC/DHPC and DPPC/ DHPC bicelles has been widely reported. ${ }^{1,2,4,5}$ The ideal bicelle model comprises a discoidal micelle with a diameter of approximately $15 \mathrm{~nm}$; although depending on their composition, lipid concentration, and long-/short-chain phospholipid molar ratio, bicelles display various morphologies. ${ }^{2,7}$ In general, bicelle size increases with the molar ratio but decreases with increasing values of lipid concentration. ${ }^{4}$ Temperature also exerts significant effects on bicelles. At temperatures higher than the long-chain phospholipid $T_{\mathrm{m}}$, bicelles undergo morphological transitions. ${ }^{1,2}$ Bicelle composition is a key factor in determining aggregate morphologies at different temperatures and in design of systems for specific conditions. Due to this morphological versatility, there is no consensus on a unique term to define the nanostructures that constitute the "bicellar system". 7 Sanders et al. ${ }^{1}$ and Triba et al. ${ }^{2}$ reported a decrease in fluidity of classical bicellar systems through temperature. Given that herein the composition of classic bicelles is altered (SCHOL replaces DHPC), the systems' transition temperature and morphology also change (as the DSC, LAURDAN GP and FFEM results show). Therefore, it is reasonable to surmise that system fluidity also changes, as a decrease in the fluidity was visually perceived.

Interestingly, the $T_{\mathrm{m}}$ decreased from $41{ }^{\circ} \mathrm{C}$ for the pure $\mathrm{DPPC}^{25}$ and DPPC/DHPC systems ${ }^{35}$ to $34{ }^{\circ} \mathrm{C}$ for DPPC/ SCHOL (molar ratio $3.5: 1$ ). For DMPC/SCHOL (molar ratio $3.5: 1)$, no temperature associated with the main lipid transition (from a gel to a liquid disordered phase) was detected; this temperature was determined to be $23{ }^{\circ} \mathrm{C}$ for pure DMPC. ${ }^{35}$ These results agree with previous findings published by $\mathrm{Le}$ Grimellec et al. ${ }^{10}$ who claim that increasing levels of SCHOL can decrease the $T_{\mathrm{m}}$ of phospholipids and even suppress it at $30-50 \%$ molar SCHOL. Herein, the SCHOL concentration is $23 \%$ molar. Thus, our evidence for the DPPC/SCHOL system is consistent with these authors, although absence of $T_{\mathrm{m}}$ for the DMPC/SCHOL system is expected for higher 
SCHOL concentrations. This has also been observed with additional sterols, where inclusion in the lipid bilayers induces formation of an extra lamellar phase, the liquid-ordered phase, which shares characteristics of both gel and fluid phases. ${ }^{9,27,30}$ Therefore, our results indicate a liquid-ordered phase for DMPC/SCHOL systems. For SCHOL, Le Grimellec et al. ${ }^{10}$ reported a fluidising effect on the lipid bilayers for $T<T_{\mathrm{m}}$ (alkyl chain order decreases) and a rigidifying effect for $T>T_{\mathrm{m}}$ (alkyl chain order increases). In other words, incorporation of SCHOL into an ordered gel phase disturbs the order of the lipid chains, but its incorporation into a disordered fluid phase increases the lipid chain order. The small transition observed at a lower temperature may be from the redistribution of SCHOL into a bilayer. In addition, DMPC/SCHOL and DPPC/SCHOL systems have membranes with higher flexibility ( $\eta=0.09$ and 0.10 , respectively) than membranes with pure DPPC (0.008, R. Pons unpublished results) or DMPC (0.077, ref. 29). This could be explained by the liquid ordered phase.

WAXS data at room temperature support these results because the Bragg distance (d) of $0.42 \mathrm{~nm}$ corresponds to a hexagonal phase for the DPPC/SCHOL system, while for pure DPPC, the $d$ value $^{36}$ is associated with a more ordered orthorhombic phase. Therefore, at room temperature $\left(T<T_{\mathrm{m}}\right)$, a certain level of disorganisation is induced by SCHOL. However, for the DMPC/SCHOL system, the WAXS results indicate certain organisation $(d=0.43 \mathrm{~nm})$ because pure DMPC above the $T_{\mathrm{m}}$ has been reported in a liquid-crystalline phase $(d=0.46 \mathrm{~nm}){ }^{37}$ This evidence is consistent with the SCHOL effect on formation of the liquid-ordered phase described previously. ${ }^{9,38,39}$ SAXS profiles for both SCHOL systems are similar except for the reflections shifted towards a lower $q$ (higher distances) for the DPPC/SCHOL system compared with the DMPC/SCHOL system. This effect is likely from the differences in alkyl chain length for DPPC (with 16 methylene groups, C16) and DMPC (C14). This is clearly observed in the electron density profiles (inset in Fig. 2 panels $\mathrm{A}$ and $\mathrm{B}$ ) where $Z_{\mathrm{H}}$ is higher for the DPPC/SCHOL system than the DMPC/SCHOL system. SAXS fits for the SCHOL systems show an average of 5 correlated bilayers with $d=52.50 \mathrm{~nm}$ for DPPC/SCHOL and $37.10 \mathrm{~nm}$ for DMPC/ SCHOL. These repetition distances are much higher than the distances reported for multilamellar membranes with pure DPPC ( $d=6.25$, R. Pons unpublished results) and DMPC $\left(d=6.34 \mathrm{~nm},{ }^{29}\right)$. Considering the $d$ equation $\left(d=d_{\mathrm{B}}+d_{\mathrm{W}}\right)$, this noted increase in $d$ may be from high interbilayer water content $\left(d_{\mathrm{w}}\right)$ through repulsion of negatively charged sulphate groups. ${ }^{40}$ In fact, $d_{\mathrm{w}}$ is much lower in multilamellar membranes with pure phospholipids (1.13 nm for pure DPPC [R. Pons unpublished results] and $1.66 \mathrm{~nm}$ for pure $\mathrm{DMPC}^{29}$ ) than for SCHOL systems. Additionally, the bilayer thickness $\left(d_{\mathrm{B}}\right)$ may contribute to increased $d$ values because $d_{\mathrm{B}}$ for SCHOL systems is higher (see Table 1) than that for pure phospholipids $(5.12 \mathrm{~nm}$ for DPPC [R. Pons unpublished results] and $4.68 \mathrm{~nm}$ for DMPC). ${ }^{29}$ Even the $d_{\mathrm{B}}$ for SCHOL systems is higher than the $d_{\mathrm{B}}$ for classical bicelles $\left(5.4 \mathrm{~nm}\right.$ for DPPC/DHPC ${ }^{32}$ and $4.5 \mathrm{~nm}$ for the DMPC/DHPC system). ${ }^{31}$ This difference may be explained by extension of the alkyl chains for incorporation of SCHOL into the bilayer. ${ }^{41}$ The distribution of SCHOL in the bilayer is asymmetric, which leads to local curvature in the membrane that corresponds to the ripple phase observed in panels $\mathrm{E}$ and $\mathrm{F}$ in Fig. 3. This undulated phase is longer than that described as the primary ripple ${ }^{42}$ or stable ripple phase, ${ }^{43}$ it is referred to as the "long ripple phase". Other authors have previously reported formation of this phase at specific temperatures and cholesterol concentrations. ${ }^{41}$

Given that SCHOL promotes formation of a liquid-ordered phase and the micrographs in Fig. 3 show large lamellar structures with and without ripple phase regions, we can assume that both SCHOL systems would form gel domains (ripple phase) and liquid-ordered domains at room temperature. These findings are consistent with the phase diagrams reported by Vist et al. ${ }^{44}$ and Meyer et al. ${ }^{38}$ for similar binary systems formed from DMPC or DPPC and cholesterol.

As for the DPPC/SCHOL system, when the temperature rises above $T_{\mathrm{m}}\left(34{ }^{\circ} \mathrm{C}\right)$, the lipids in the gel phase domains (SCHOL-poor domains) melt to the fluid phase, while liquidordered domains are unaltered (SCHOL-rich domains), which explains why an asymmetric main transition temperature is observed in DSC. The absence of a measurable $T_{\mathrm{m}}$ for the DMPC/SCHOL system indicates the predominant presence of a liquid-ordered phase, whereas SCHOL distribution in the bilayer could be more uniform than for DPPC/SCHOL because miscibility of sterol decreases with increasing alkyl chain length in the phospholipid. ${ }^{45}$ This SCHOL uniform distribution likely undergoes a transition from a ripple to liquid-ordered phase. This transition would not involve significant conformational changes and thus could not be detected by DSC. ${ }^{46}$

\section{Skin application of the systems}

Contact of these systems with the skin involves a variation in their temperature from room to skin temperature $\left(22\right.$ to $\left.32^{\circ} \mathrm{C}\right)$. In this situation, certain nanostructures in the systems can undergo morphological changes due to the different phospholipid $T_{\mathrm{m}}$. For instance, the DMPC/DHPC system has a $T_{\mathrm{m}}$ of $23^{\circ} \mathrm{C}$, and as a consequence, when in contact with the skin at $32{ }^{\circ} \mathrm{C}$, the system state changes from a gel to fluid phase, and the nanostructures in the system increase in size, as described previously. ${ }^{34}$ These large structures cannot penetrate intact into the narrow interlamellar spaces of the SC and are principally located at the skin surface. It is likely that these systems partially disaggregate through the effects of hydration and temperature on the skin, and separated lipid molecules can penetrate beyond the superficial layers of the skin (see ATR-FTIR results ${ }^{47}$ ). Our findings are consistent with this assumption because the IR experiments (see the Results section) demonstrated that the perdeuterated phospholipids formed in the DMPC/DHPC system are detected in the deep layers of skin, although the effects from this system are greater at the superficial layers (see the $\mathrm{CH}_{2}$ stretching and $\Delta \mathrm{GP}$ experiments). Given that no phase transition was detected for the DMPC/SCHOL system, significant morphological changes are unexpected after application on the skin through a change in temperature from $22{ }^{\circ} \mathrm{C}$ to $32{ }^{\circ} \mathrm{C}$. The large structures that form the SCHOL systems (see Fig. 3A and B) could disaggregate and form smaller structures that can penetrate the skin with the small structures already present in this system. This process, the increase in membrane flexibility in SCHOL systems and the compatibility of SCHOL with the skin, 
could increase their penetration into the skin (see the ATR-FTIR results for the perdeuterated systems). The DPPC/DHPC system has a $T_{\mathrm{m}}$ at $41{ }^{\circ} \mathrm{C}$; thus, when the system is applied to the skin at $32{ }^{\circ} \mathrm{C}$, it is in the gel phase. Given that no phase changes are produced, modifications in size and morphology were unexpected. The DPPC/DHPC system is formed by small structures, as reported in previous work. ${ }^{4}$ These small structures can penetrate the skin in a more homogeneous manner (see the ATR-FTIR results). The DPPC/SCHOL system has a $T_{\mathrm{m}}$ at $34^{\circ} \mathrm{C}$; after application to the skin, this system has SCHOL-rich domains in a liquid-ordered phase and SCHOLpoor domains in transition between a ripple and fluid phase. Considering that SCHOL has a fluidising effect on the system under these conditions and given the compatibility of SCHOL with the aforementioned skin lipids, this system may penetrate deep into the skin (see the ATR-FTIR results).

In summary, these systems in contact with the skin exhibit different phases with the lipid order decreasing as follows.

\section{DPPC/DHPC (gel phase) > DPPC/SCHOL (transition ripple/fluid + liquid-ordered) $\simeq \mathrm{DMPC} / \mathrm{SCHOL}$ (liquid-ordered) $>$ DMPC/DHPC (fluid phase)}

Taking into account this thermotropic behaviour, the effect of these systems on SC lipids is different. The DPPC systems (with a more ordered structure) modify SC lipid organisation less than the DMPC systems. Concerning the effect of topical applications with components in a fluid state, the FTIR spectroscopy ${ }^{48}$ and TPE microscopy ${ }^{7,49}$ results for skin treated with oleic acid or dioleoylphosphatidylcholine support this conclusion. Additionally, Van den Bergh et al. ${ }^{50}$ reported that the elastic vesicles or transfersomes formed from surfactants/ lipid induce a penetration-enhancing effect.

This thermotropic behaviour is also related to structure stability and hence the distribution of the system into skin. DMPC systems should be more unstable structures in contact with the skin (due to their fluid state) than the DPPC systems (in a gel state). Thus, DMPC systems could disaggregate at the superficial skin layers, while DPPC systems should diffuse deeper into the skin. The following factors could be related to the high accumulation of SCHOL in certain skin layers. (1) This lipid is a component of the SC, thus, the interchange between components of the system and SC lipids could be conceived. (2) SCHOL contributes membrane stability, ${ }^{51}$ and (3) SCHOL systems have small structures (see Fig. 3C and D) that could penetrate intact into skin.

On the other hand, sterol treatment of perturbed skin improves skin barrier function. ${ }^{52}$ Although, our recent works have demonstrated that DHPC bicelles are good for skin applications, ${ }^{3,4}$ the use of SCHOL in these new systems could be better for dermatological applications. This is because SCHOL is a component of skin and it is thus more compatible with the skin than DHPC.

Currently, chemical and physical skin penetration enhancers are employed by the pharmaceutical and the cosmetic industry, which, in certain cases, are associated with irritating and toxic effects. ${ }^{53}$ The systems with SCHOL proposed herein do not modify the SC lipid organisation as the results from IR and GP
LAURDAN demonstrated; further, these systems may act as lipid reservoirs for the skin. For these reasons, use of bicellar systems with SCHOL may be an interesting strategy for effective drug delivery across the skin in the same way that classic bicelles with DHPC are used to incorporate drugs into their bilayer. $^{35}$

\section{Acknowledgements}

The authors wish to thank Jaume Caelles from SAXS/WAXS service at IQAC, Josep Carrilla, and Rocío Vicente from Microcalorimetry service at IQAC. M. Cócera is funded by the JAE-DOC program from CSIC (co-funded by FSE). This work was supported by funds from CICYT (CTQ 2010-16964) and from Generalitat de Catalunya (2009 SGR 1212). The LAURDAN generalized polarization experiments, both imaging and cuvette, and their analysis have been performed at the Danish Molecular Bioimaging Center (DaMBIC), University of Southern Denmark, Odense, Denmark.

\section{References}

1 C. R. Sanders 2nd and J. P. Schwonek, Biochemistry, 1992, 31, 8898-8905.

2 M. N. Triba, D. E. Warschawski and P. F. Deveaux, Biophys. J., 2005, 88, 1887-1901.

3 L. Barbosa-Barros, C. Barba, G. Rodríguez, M. Cócera, L. Coderch, C. López-Iglesias, A. de la Maza and O. López, Mol. Pharmaceutics, 2009, 6, 1237-1245.

4 L. Barbosa-Barros, G. Rodríguez, C. Barba, M. Cócera, L. Rubio, J. Estelrich, C. López-Iglesias, A. de la Maza and O. López, Small, 2012, 8, 807-818.

5 L. V. Dam, G. Karlsson and K. Edwards, Langmuir, 2006, 28, $3280-3285$.

6 R. Soong and P. M. Macdonald, Langmuir, 2009, 25, 380-390.

7 G. Rodríguez, M. Cócera, L. Rubio, C. López-Iglesias, R. Pons, A. de la Maza and O. López, Mol. Pharmaceutics, 2012, 9, 482-491.

8 C. A. Strott and Y. Higashi, J. Lipid Res, 2003, 44, 1268-1278.

9 M. Eeman and M. Deleu, Biotechnol., Agron., Soc. Environ., 2010, 14, 719-736.

10 C. Le Grimellec, A. Daigneault, G. Bleau and K. D. Roberts, Lipids, 1984, 19, 474-477.

11 M. Boncheva, F. Damien and V. Normand, Biochim. Biophys. Acta, 2008, 1778, 1344-1355.

12 I. Plasencia, L. Norlén and L. A. Bagatolli, Biophys. J., 2007, 93, $3142-3155$.

13 R. A. Videira, M. C. Antunes-Madeira and V. M. Madeira, Biochim. Biophys. Acta, 1999, 1419, 151-163.

14 T. Parasassi, G. De Stasio, A. d'Ubaldo and E. Gratton, Biophys. J., 1990, 57, 1179-1186.

15 D. Orthaber, A. Bergmann and O. Glatter, J. Appl. Crystallogr., 2000, 33, 218-225.

16 L. Pérez, A. Pinazo, M. R. Infante and R. Pons, J. Phys. Chem. B, 2007, 111, 11379-11387.

17 J. S. Pedersen, Adv. Colloid Interface Sci., 1997, 70, 171-210.

18 G. Pabst, M. Rappolt, H. Amenitsch and P. Laggner, Phys. Rev. E, 2000, 62, 4000-4009.

19 B. Papahadjopoulos-Sternberg, Methods Mol. Biol., 2010, 606, 333-349.

20 C. Brancewicz, D. H. Rasmussen and B. Papahadjopoulos-Sternberg, J. Disp. Sci. Technol., 2006, 27, 761-765.

21 S. U. Egelhaaf, E. Wehrli, M. Muller, M. Adrian and P. Schurtenberger, J. Microsc. (Oxford, U. K.), 1996, 184, 214-228.

22 H. Wagner, K.-H. Kostka, C.-M. Lehr and U. F. Schaefer, Pharm. Res., 2000, 17, 1475-1481.

23 V. P. Shah, G. L. Flynn, A. Yacobi, H. I. Maibach, C. Bon, N. M. Fleischer, T. J. Franz, S. A. Kaplan, J. Kawamoto, L. J. Lesko, J.-P. Marty, L. K. Pershing, H. Schaefer, J. A. Sequeira, 
S. P. Shrivastava, J. Wilkin and R. L. Williams, Pharm. Res., 1998, 15, 167-171.

24 J. Brewer, J. Bernardino de la Serna, K. Wagner and L. A. Bagatolli, Biochim. Biophys. Acta, 2010, 1798, 1301-1308.

25 J. Sabin, G. Prieto, J. Estelrich, F. Sarmiento and M. Costas, J. Colloid Interface. Sci., 2010, 348, 388-392.

26 A. B. Hendrich, O. WesoLowska and K. Michalak, Biochim. Biophys. Acta, 2001, 1510, 414-425.

27 R. Wu, L. Chen, Z. Yu and P. J. Quinn, Biochim. Biophys. Acta, 2006, 1758, 764-771.

28 H. Yoshida, T. Hatakeyama and H. Hatakeyama, J. Therm. Anal. Calorim., 1992, 40, 483-489.

29 G. Pabst, J. Katsaras, V. A. Raghunathan and M. Rappolt, Langmuir, 2003, 19, 1716-1722.

30 L. Chen, Z. Yu and P. J. Quinn, Biochim. Biophys. Acta, 2007, 1768, 2873-2881.

31 L. Barbosa-Barros, C. Barba, M. Cócera, L. Coderch, C. López-Iglesias, A. de la Maza and O. López, Int. J. Pharm., 2008, 352, 263-272.

32 L. Barbosa-Barros, A. de la Maza, J. Estelrich, A. M. Linares, M. Feliz, P. Walther, R. Pons and O. López, Langmuir, 2008, 24, 5700-5706.

33 L. Coderch, M. de Pera, N. Perez-Cullell, J. Estelrich, A. de la Maza and J. L. Parra, Skin Pharmacol. Appl. Skin Physiol., 1999, 12, 235-246.

34 G. Rodríguez, L. Barbosa-Barros, L. Rubio, M. Cócera, A. Diez, J. Estelrich, R. Pons, J. Caelles, A. de la Maza and O. López, Langmuir, 2009, 25, 10595-10603.

35 L. Rubio, G. Rodríguez, C. Alonso, C. López-Iglesias, M. Cócera, L. Coderch, A. de la Maza, J. L. Parra and O. López, Soft Matter, 2011, 7, 8488-8497.

36 S. D. Guler, D. D. Ghosh, J. Pan, J. C. Mathai, M. L. Zeidel, J. F. Nagle and S. Tristram-Nagle, Chem. Phys. Lipids, 2009, 160, 33-44.
37 J. A. Clarke, A. J. Heron, J. M. Seddon and R. V. Law, Biophys. J., 2006, 90, 2383-2393.

38 F. J. de Meyer, A. Benjamini, J. M. Rodgers, Y. Misteli and B. Smit, J. Phys. Chem. B, 2010, 114, 10451-10461.

39 J. M. Holopainen, J. Lemmich, F. Richter, O. G. Mouritsen, G. Rapp and P. K. J. Kinnunen, Biophys. J., 2000, 78, 2459-2469.

40 C. Faure, J. F. Tranchant and E. J. Dufourc, Biophys. J., 1996, 70, $1380-1390$.

41 S. Karmakar, Thesis, Jawaharlal Nehru University, 2005.

42 L. Dahbi, M. Arbel-Haddad, P. Lesieur, C. Bourgaux and M. Ollivon, Chem. Phys. Lipids, 2006, 139, 43-53.

43 C. Leidy, T. Kaasgaard, J. H. Crowe, O. G. Mouritsen and K. Jorgensen, Biophys. J., 2002, 83, 2625-2633.

44 M. R. Vist and J. H. Davis, Biochemistry, 1990, 29, 451-464.

45 N. Borochov, E. J. Wachtel and D. Bach, Chem. Phys. Lipids, 1995, 76, 85-92.

46 G. M'Baye, Y. Mely, G. Duportail and A. S. Klymchenko, Biophys. $J ., 2008,95,1217-1225$.

47 G. Rodríguez, L. Rubio, M. Cócera, J. Estelrich, R. Pons, A. de la Maza and O. López, Langmuir, 2010, 26, 10578-10584.

48 M. Cotte, P. Dumas, M. Besnard, P. Tchoreloff and P. Walter, J. Controlled Release, 2004, 97, 269-281.

49 Y. Sun, W. Lo, S. J. Lin, S. H. Jee and C. Y. Dong, Opt. Lett, 2004, 29, 2013-2015.

50 B. A. I. van den Bergh, J. Vroom, H. Gerritsen, H. E. Junginger and J. A. Bouwstra, Biochim. Biophys. Acta, 1999, 1461, 155-173.

51 O. López, M. Cócera, A. de la Maza, L. Coderch and J. L. Parra, Biochim. Biophys. Acta, 2000, 1508, 196-209.

52 M. Loden and A. C. Andersson, Br. J. Dermatol., 1996, 134, 215-220.

53 L. T. Fox, M. Gerber, J. D. Plessis and J. H. Hamman, Molecules, 2011, 16, 10507-10540. 